\title{
Das lange Leben lernen
}

\section{ZfE}

\author{
James W. Vaupel · Dirk Hofäcker
}

Zusammenfassung: Die Wahrscheinlichkeit, dass Menschen ein hohes Alter erreichen, ist im vergangenen Jahrhundert in den industrialisierten Ländern erheblich gestiegen. Sinkende Geburten- und Sterberaten haben zu einer veränderten Bevölkerungsstruktur geführt. In den kommenden Jahrzehnten wird die Bevölkerung in Deutschland rasch altern. Diese neue demografische Lage erfordert Anpassungen und Umdenken auf persönlicher und sozialpolitischer Ebene. Wir argumentieren, dass die Förderung von Bildung über alle Lebensphasen und Bevölkerungsgruppen hinweg im Zentrum der Anpassungsstrategien stehen muss. Das gilt beispielsweise in Bezug auf Gesundheit, denn künftige Belastungen der sozialen Sicherungssysteme und die gesamtwirtschaftliche Leistungsfähigkeit hängen auch davon ab, in welchem Gesundheitszustand die wachsende Zahl älterer Menschen sein wird. Bildung kann ein gesundheitsförderndes Verhalten unterstützen und damit die Chancen erhöhen, dass ein Mensch ein langes, aktives Leben führen kann. Zudem kommen Aus- und Weiterbildung durch den demografischen Wandel eine noch höhere Bedeutung im Arbeitsleben zu, da durch die Veränderungen in der Bevölkerungsstruktur eine Verlängerung der Lebensarbeitszeit und eine gleichmäßigere Verteilung der Arbeit über den Lebenslauf notwendig werden. Bildung ist eine Investition, die sich auch und gerade bei der Aussicht auf ein langes Leben lohnt und das Fundament bildet, auf dem Menschen ihr Leben eigenverantwortlich führen, sich persönlich und beruflich entwickeln und ihren Lebensunterhalt sichern können.

Schlüsselwörter: Langlebigkeit · Lebenserwartung · Demografischer Wandel · Bildung · Arbeit

\section{Learning to live long}

\begin{abstract}
In the industrialized world the probability of people reaching higher ages has risen substantially over the past decade. Falling birth and mortality rates have led to changes in the population structure. In Germany, the population will be aging rapidly over the next (decades), with the new demographic situation necessitating adjustment and re-orientation on the individual and sociopolitical level. We argue that the adjustment strategies should focus on the promotion of education across all phases of life and population groups. This applies to the issue of health, for example,
\end{abstract}

\footnotetext{
Online publiziert: 03.11.2009

Prof. J. W. Vaupel, Ph. D. ( $₫)$

Max-Planck-Institut für demografische Forschung, Konrad-Zuse-Str. 1

18057 Rostock, Deutschland

E-Mail: jwv@demogr.mpg.de

Dr. D. Hofäcker

Staatsinstitut für Familienforschung an der Universität Bamberg (ifb)

Heinrichsdamm 4, 96047 Bamberg, Deutschland

E-Mail: dirk.hofaecker@ifb.uni-bamberg.de
}

(C) Die Autoren 2009. Dieser Artikel ist auf Springerlink.com mit Open Access verfügbar. 
since future strains on the social welfare system and macroeconomic performance are affected, among other factors, by the state of health of the growing number of elderly people. Education boosts health-promoting behavior, thus increasing the chances of an individual enjoying a long, active life. Besides, educational training and professional development are gaining importance owing to demographic change as the changes in the population structure will require individuals to work longer over their working lives and lead to a more even distribution of gainful employment across the life course. Education is a worthwhile investment, in particular as the prospects for long life are rising and because it enables people to lead their lives as responsible agents, to take charge of their personal individual and professional development, and to assure their livelihood.

Keywords: Demographic change $\cdot$ Education $\cdot$ Longevity $\cdot$ Life expectancy $\cdot$ Work

\section{Einleitung}

Im Laufe des 20. Jahrhunderts ist die Lebenserwartung in Deutschland um etwa 30 Jahre gestiegen. Sollte die Sterblichkeit weiterhin abnehmen wie bisher, hat ein Kind, das heute in Deutschland geboren wird, eine Chance von mehr als 50 Prozent, seinen 100. Geburtstag feiern zu können. Dass die durchschnittliche Lebenserwartung zumindest in den westlichen Industriestaaten kontinuierlich gestiegen ist, stellt eine der größten Errungenschaften moderner Gesellschaften dar. Gleichzeitig sind hiermit große Herausforderungen verknüpft, denn die demografische Entwicklung der vergangenen Jahrzehnte hat bereits grundlegende Änderungen in der Bevölkerungsstruktur verursacht. Dabei ging die steigende Lebenserwartung mit niedrigen Geburtenraten und einer geringen Nettomigration einher. Diese Bevölkerungsentwicklung wird vermutlich für einige Jahrzehnte unumkehrbar sein.

Die teilweise weitreichenden Folgen dieses demografischen Wandels haben in jüngerer Vergangenheit vermehrt Eingang in die wissenschaftliche und die öffentliche Debatte gefunden:

(i) Mit dem Anstieg der Lebenserwartung ist die Frage verbunden, ob der Zugewinn an Lebensjahren mit einem Altern in Gesundheit ohne körperliche Beschwerden oder mit längeren Krankheits- und Pflegephasen verbunden sein wird. Daraus folgt die Frage, wie in Zukunft die Pflegeleistungen für die ältere Bevölkerung gestaltet und finanziert werden können.

(ii) Aufgrund der zu erwartenden Alterung der Bevölkerung und einem gleichzeitigen Trend zur Frühverrentung sehen sich Sozialversicherungs- und Rentensysteme in modernen Gesellschaften zunehmend mit einem Ungleichgewicht zwischen Beitragszahlern und Transferempfängern konfrontiert (Hofäcker, in press; World Bank 1994).

(iii) Wenn die Wahrscheinlichkeit steigt, ein langes Leben führen zu können, wird dies auch Konsequenzen für die Lebensplanung des Einzelnen sowie für seine Bildungs-, Erwerbs- und Berufsbiografie haben. Vor diesem Hintergrund stellt sich die Frage, inwiefern eine am klassischen, chronologisch strukturierten „Standardlebenslauf“ (Kohli 1985) orientierte Sozialpolitik zukünftig in der Lage sein wird, soziale Sicherheit und gerechte Umverteilung zwischen und innerhalb von Generationen zu gewährleisten. 
Dieser hier kursorisch vorgenommene Überblick von möglichen Problemstellungen verdeutlicht, dass die Bevölkerungsalterung, die aus einem Zusammenspiel von steigender Lebenserwartung und sinkenden Geburtenraten resultiert, in zunehmendem Maße innovative Anpassungen und ein Umdenken auf persönlicher und sozialpolitischer Ebene erfordern wird. Aus verschiedenen sozial- und wirtschaftswissenschaftlichen Disziplinen liegen hierzu Reformvorschläge vor. Der vorliegende Beitrag ergänzt die gegenwärtige Debatte um die These, dass bei der Bewältigung des demografischen Wandels Bildung eine entscheidende Rolle spielen kann: Bildung stellt unter den Bedingungen des gegenwärtigen demografischen und sozioökonomischen Wandels einen wesentlichen Aspekt einer umfassenden Anpassungsstrategie für ein langes, gesundes und produktives Leben dar.

Für den Einzelnen bedeutet Bildung eine Investition, die bei höherer Lebenserwartung über einen längeren Zeitraum gewinnbringend genutzt werden kann (Lee \& Goldstein 2003). Dabei wird es durch die Verlängerung der individuellen Lebensspanne zunehmend erforderlich, sein Wissen kontinuierlich aufzufrischen und obsoletes Wissen durch neue Inhalte $\mathrm{zu}$ ersetzen - insbesondere in Zeiten rapiden technologischen und gesellschaftlichen Fortschritts (vgl. Blossfeld 2003; vbw 2008).

Darüber hinaus vermag Bildung gesamtgesellschaftlich dazu beizutragen, die Herausforderungen des demografischen Wandels besser zu bewältigen. Diese zentrale These soll im Folgenden näher erläutert werden. Kapitel 1 beschreibt als Ausgangspunkt grundlegende Bevölkerungstrends der vergangenen Jahrzehnte; Kapitel 2 skizziert die hierdurch entstehenden Herausforderungen und Anpassungsprobleme und verdeutlicht, inwiefern Bildung zu deren Bewältigung beitragen kann; Kapitel 3 fasst schließlich die Ergebnisse zusammen und weist auf Konsequenzen für künftige politische Weichenstellungen und für die sozialwissenschaftliche Forschung hin.

\section{Alterndes Deutschland: Der demografische Wandel}

Die Bevölkerungsalterung ist ein globaler Prozess, in dem die industrialisierten Länder am weitesten fortgeschritten sind. Vor allem in Europa und Japan wird sich die Alterung besonders beschleunigen (Lutz et al. 2008). Schon heute ist Deutschland - gemessen am Anteil der über 60-Jährigen an der Gesamtbevölkerung - nach Japan und Italien das drittälteste Land der Welt. Den Prognosen der Vereinten Nationen zufolge wird sich an der Spitzenstellung dieser drei Länder bis zur Mitte des 21. Jahrhunderts wenig ändern (United Nations 2007).

Immer mehr ältere Menschen werden in den kommenden Jahrzehnten somit immer weniger jungen Menschen gegenüberstehen. Lag der Anteil der über 65-Jährigen an der Gesamtbevölkerung Deutschlands 2005 bei 19\%, so geht das Statistische Bundesamt (2006) in seiner relativ vorsichtigen Vorausberechnung ${ }^{1}$ davon aus, dass sich dieser Wert bis 2030 auf knapp 30\% und bis 2050 auf 33\% der Gesamtbevölkerung erhöhen wird. Während die sogenannten „Babyboomer“, d.h. die geburtenstarken Jahrgänge der 1950er- und 1960er-Jahre, heute noch im mittleren Erwerbsalter sind, stellen sie schon bald die Gruppe der älteren Erwerbstätigen und Transferempfänger, was eine nachhaltige Verschiebung des Verhältnisses von Rentnern zu Menschen im Erwerbsalter nach sich 
ziehen wird. Während heute noch drei Personen im erwerbsfähigen Alter auf einen Menschen im Rentenalter kommen, werden es im Jahr 2030 nur noch zwei sein.

Kausal lässt sich der demografische Wandel insbesondere auf zwei gegenwärtig parallel stattfindende demografische Prozesse zurückführen: (i) den kontinuierlichen Anstieg der Lebenserwartung sowie (ii) die dauerhaft niedrigen Geburtenraten unterhalb des so genannten Bestandserhaltungsniveaus.

\subsection{Lebenserwartung und Langlebigkeit}

Seit Mitte des 19. Jahrhunderts hat die Lebenserwartung in den Industriegesellschaften stetig zugenommen (Oeppen \& Vaupel 2002). In den Ländern, die in dem jeweiligen Jahr die weltweit höchste Lebenserwartung bei Frauen hatten, ist in den vergangenen anderthalb Jahrhunderten ein linearer Anstieg der Lebenserwartung von fast drei zusätzlichen Lebensmonaten pro Jahr festzustellen (vgl. Abb. 1). Waren 1840 noch die Schwedinnen

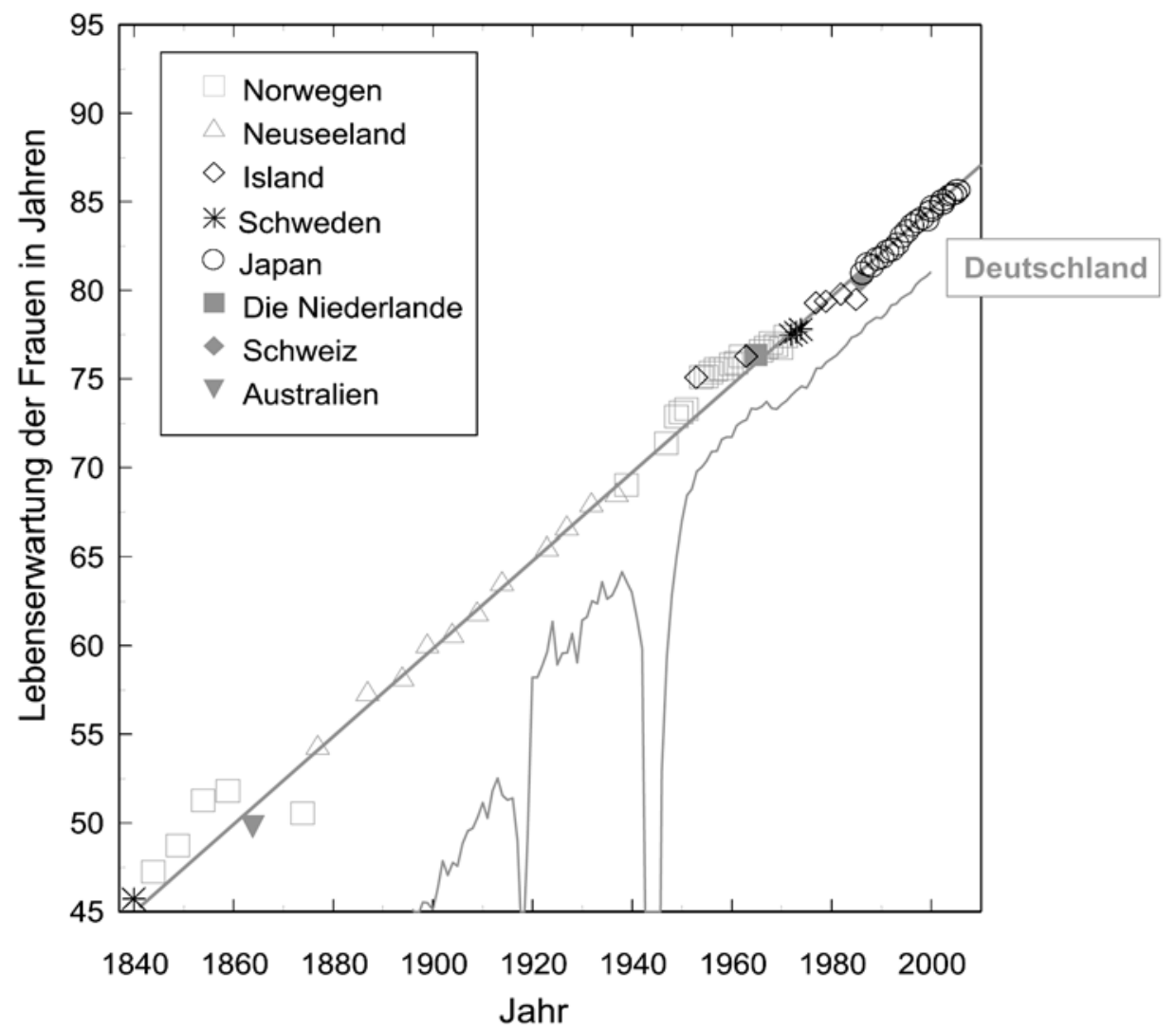

Abb. 1: Lebenserwartung der Frauen von 1840 bis zur Gegenwart in dem jeweils rekordhaltenden Land, gekennzeichnet durch Symbole, und separat in Deutschland. Die Regressionsgerade der rekordhaltenden Länder hat eine Steigung von 0,243. Adaptiert nach Oeppen \& Vaupel 2002. 
mit einer Lebenserwartung von 45 Jahren die Spitzenreiterinnen, so sind es heute mit 86 Jahren die Japanerinnen (PRB 2008). Auch in Deutschland steigt die Lebenserwartung seit Mitte der 1950er-Jahre an, wobei der Abstand zum Rekordhalter konstant drei bis vier Jahre beträgt. Blickt man bis ans Ende des 19. Jahrhunderts zurück, so waren die Verbesserungen in der Überlebenswahrscheinlichkeit im ersten Lebensjahr besonders groß: 1871 starb in Deutschland jeder vierte Säugling im ersten Lebensjahr und 1900 noch jeder fünfte vor Erreichen des ersten Lebensjahrs. Heute feiern 996 von 1000 Lebendgeborenen ihren ersten Geburtstag.

Die Wahrscheinlichkeit zu sterben hat jedoch nicht nur im Kleinkindalter, sondern in jedem Lebensalter über die Jahre hinweg abgenommen, wobei Frauen durchschnittlich länger als Männer leben (Barford et al. 2006; Doblhammer 2006). Dennoch ist auch die Lebenserwartung der Männer seit 1840 nahezu linear gestiegen, und das nur unwesentlich langsamer als die der Frauen. Während die Lebenserwartung in Deutschland zwischen 1901 und 1910 für Männer noch 44,8 und für Frauen 48,3 Jahre betrug, ${ }^{2}$ liegt sie heute nach der Sterbetafel 2005/2007 des Statistischen Bundesamtes für Männer bei 76,9 und für Frauen bei 82,3 Jahren. Die Ursachen für das längere Überleben liegen in einem Zusammenspiel verschiedener gesellschaftlich induzierter Faktoren, zu denen steigender Wohlstand, gesunde Ernährung, bessere Arbeitsbedingungen mit zunehmend geringerer körperlicher Belastung sowie verbesserte Hygiene, medizinische Versorgung und soziale Fürsorge gehören (Riley 2001).

Infolge des Anstiegs der Lebenserwartung wächst die Bevölkerungsgruppe der hochbetagten Personen rasch (Rau et al. 2006). Immer mehr Menschen erreichen ein Alter von 90 oder 100 Jahren - vor noch nicht langer Zeit war das ein sehr seltenes Ereignis. Die Analyse der Hochbetagtenstatistiken seit 1960 zeigt eine jährliche Zuwachsrate zwischen 5 und $11 \%$ in ausgewählten industrialisierten Ländern, wobei Japan den Spitzenwert erreicht (vgl. Tab. 1). Im Laufe des Jahres 2004 konnten in Deutschland über 10.000 Menschen ihren 100. oder einen höheren Geburtstag feiern. Gegenüber dem Jahr 1960 bedeutet dies eine Steigerung um das 45 -fache.

Weder der Trend der Rekord-Lebenserwartung noch die unterschiedlichen Verläufe der Entwicklung in einzelnen Ländern legen ein baldiges Ende dieses Anstiegs der Lebenserwartung nahe (Oeppen \& Vaupel 2002). Zwar folgen in einzelnen Ländern typischer-

Tab. 1: Anzahl der Menschen über 80 Jahre, über 90 Jahre und über 100 Jahre in ausgewählten industrialisierten Ländern in den Jahren 1960 und 2000

\begin{tabular}{lrrrrrr}
\hline & \multicolumn{1}{l}{ Bevölkerung 1960 im Alter } & \multicolumn{3}{c}{ Bevölkerung 2004 im Alter } \\
\hline D0+ & \multicolumn{1}{c}{$\mathbf{9 0 +}$} & $\mathbf{1 0 0 +}$ & $\mathbf{8 0}+$ & \multicolumn{1}{c}{$\mathbf{9 0}+$} & \multicolumn{1}{c}{$\mathbf{1 0 0 +}$} \\
\hline Dänemark & 81,542 & 5,713 & 36 & 234,641 & 39,077 & 801 \\
Deutschland & $1,276,932$ & 64,789 & 229 & $3,615,233$ & 598,827 & 10,243 \\
Japan & 720,166 & 36,022 & 199 & $6,303,724$ & $1,126,303$ & 29,433 \\
Norwegen & 76,290 & 7,284 & 102 & 224,826 & 33,334 & 646 \\
Spanien & 412,918 & 36,336 & 599 & $1,941,947$ & 302,211 & 6,912 \\
Schweden & 152,380 & 11,172 & 95 & 508,872 & 81,580 & 1,607 \\
Schweiz & 89,600 & 5,450 & 39 & 344,497 & 62,088 & 1,183 \\
\hline
\end{tabular}

Quelle: Berechnungen Roland Rau, MPIDR. Daten: Kannisto-Thatcher Database on Old Age Mortality. 
weise auf Phasen des raschen Aufholens langsamere Anstiege der Lebenserwartung, doch nähern sich die Kurven keineswegs asymptotisch maximalen Werten an. Gerade in Ländern mit den höchsten Werten der Lebenserwartung, wie Japan, Frankreich und Schweden, ist kein Anzeichen eines Abflachens dieses Trends auszumachen.

Prognosen, die den linearen Trend aufnehmen, stützen sich auf Zeitreihenanalysen. Diesen zufolge wäre in Deutschland im Jahr 2050 mit einer Lebenserwartung von deutlich über 90 Jahren zu rechnen ( $94 \pm 2,8$ Jahre; Vaupel et al. 2006). Sowohl das Statistische Bundesamt als auch Eurostat bleiben mit ihren Prognosen vorsichtiger, weil sie davon ausgehen, dass das Potenzial zur Verringerung der Alterssterblichkeit bereits weitgehend ausgeschöpft ist und der Anstieg der Lebenserwartung entsprechend langsamer verlaufen wird als in den vergangenen Jahren. Bis 2050 erwartet das Statistische Bundesamt einen Anstieg der Lebenserwartung bei Frauen auf 88 Jahre (niedrige) bzw. 89,9 Jahre (höhere Variante).

Schon ein geringer Anstieg der Lebenserwartung bewirkt jedoch eine deutliche Zunahme an Hochbetagten, deren Versorgung, Betreuung und Pflege die Gesellschaft vor neue Herausforderungen stellen werden. Natürlich kann niemand die Zukunft genau vorhersagen. Zu gering angesetzte Prognosen könnten aber fatale Folgen haben: Sie könnten dazu beitragen, dass das Ausmaß der Alterung der Gesellschaft ebenso wie die Verschiebung des Verhältnisses von Beitragszahlern zu Rentenbeziehern unterschätzt und damit Reformen möglicherweise zu spät ergriffen werden.

\subsection{Geburtenentwicklung}

Den zweiten zentralen Aspekt des demografischen Wandels stellen die seit Jahrzehnten niedrigen Geburtenraten dar. Die Bevölkerung altert nicht nur, weil immer mehr Menschen ein sehr hohes Alter erreichen, sondern auch weil immer weniger junge Menschen nachrücken. Niedrige Fertilität ist gegenwärtig in den meisten industrialisierten Ländern zu beobachten. Anfang dieses Jahrhunderts war die zusammengefasste Geburtenziffer (total fertility rate, TFR) in allen europäischen Ländern unter das Niveau von 2,1 Kindern pro Frau gefallen, das notwendig ist, damit eine Kindergeneration die jeweilige Elterngeneration zahlenmäßig ersetzen kann (Eurostat 2007). Heute wohnen bereits drei Viertel der europäischen Bevölkerung in Ländern, in denen sehr niedrige zusammengefasste Geburtenziffern von weniger als 1,5 zu beobachten sind (Frejka \& Sobotka 2008). Die Ursachen für das niedrige Fertilitätsniveau in Europa sind komplex und bislang nur partiell verstanden (Sobotka 2004; Neyer \& Andersson 2004; Frejka \& Sobotka 2008).

Deutschland ist im europäischen Vergleich ein Land mit sehr niedriger Fertilität. Die durchschnittliche Geburtenziffer ging in Deutschland bereits gegen Ende der 1960erJahre zurück und liegt in den alten Bundesländern seit Mitte der 1970er-Jahre mit knapp 1,4 auf einem konstant niedrigen Niveau. In Ostdeutschland weist die Geburtenentwicklung stärkere Schwankungen auf. Auch hier sank das Geburtenniveau in den 1970erJahren, erholte sich jedoch wieder aufgrund von familienpolitischen Maßnahmen der DDR. In und nach den Wendejahren 1989 und 1990 sanken die Geburtenzahlen auf sehr niedrige Werte, was auch dadurch bedingt war, dass Frauen etwaige Geburten aufgeschoben haben. Das Absinken des Geburtenniveaus ging in beiden Teilen Deutschlands mit einem Rückgang der Zahl der Mehrkindfamilien einher sowie in Westdeutschland auch 
mit einem im internationalen Vergleich hohen Anstieg der Kinderlosigkeit (Konietzka \& Kreyenfeld 2007).

Obwohl sich die Menschen in Ost- und in Westdeutschland wie in anderen europäischen Ländern mehr Kinder für ihre eigene Familie wünschen als tatsächlich geboren werden (Dorbritz et al. 2005), ist davon auszugehen, dass die Geburtenraten zumindest vorerst auf niedrigem Niveau verbleiben werden (Frejka \& Sobotka 2008). Aufgrund des im internationalen Vergleich früh stark gesunkenen Geburtenniveaus, zumindest in Westdeutschland, ist bereits heute die Zahl der Menschen, die potenziell die Elterngeneration stellen könnten, kleiner geworden. Deshalb ist auch in den kommenden Jahren und Jahrzehnten eine geringe absolute Anzahl an Geburten in Deutschland zu erwarten - auch wenn die Geburtenrate, gemessen als TFR, konstant bleiben sollte. Die Alterung der Bevölkerung wird sich also vorerst eher noch beschleunigen als verlangsamen. ${ }^{3}$

\section{Anpassungen an den demografischen Wandel}

Aus der dargestellten Bevölkerungsentwicklung ergibt sich politischer Handlungsbedarf: Die sozialen Sicherungssysteme müssen an die neuen demografischen Gegebenheiten angepasst werden, da sich der Arbeitsmarkt auf eine alternde Erwerbsbevölkerung einstellen muss und der Pflegebedarf in den kommenden Jahren vermutlich steigen wird.

Dabei gilt zu beachten, dass demografische Prozesse vergleichsweise träge sind. Im Gegensatz zu sehr empfindlichen Konjunkturentwicklungen gehorchen Bevölkerungsentwicklungen Regelmäßigkeiten, die kurzfristige Schwankungen zwar nicht ausschließen, diese aber doch innerhalb bestimmter Grenzen halten (Vaupel et al. 2006). Zumindest kurzfristige Entwicklungen von 30 bis 50 Jahren können mit relativ großer Sicherheit vorausberechnet werden. Bei längeren Zeithorizonten muss hingegen mit größeren Unsicherheiten und weniger verlässlichen Aussagen gerechnet werden (Vaupel 2006). Trotz dieser Vorhersageunsicherheit sind demografische Prognosen für die politische Praxis unentbehrliche Instrumente, um wahrscheinliche Veränderungen vorzuzeichnen und mögliche Auswirkungen auf verschiedene Politikbereiche abzuschätzen.

Demografische Prognosen sind insbesondere deswegen unabdingbar, da entsprechende Reformen künftigen demografischen Veränderungen vorauseilen müssen, um zu gegebener Zeit ihre Wirkungen entfalten zu können. ${ }^{4}$ Hinsichtlich der künftig notwendigen Anpassungen verdienen zwei Bereiche besondere Aufmerksamkeit: die Gesundheit im hohen Lebensalter sowie die Aufteilung der Erwerbsarbeit im Lebenslauf.

Es gilt also zum Einen abzuschätzen, inwiefern die zunehmende Alterung der Bevölkerung mit einer verlängerten Phase physischer und psychischer Gesundheit einhergeht. Wie hoch wird der Anteil derer sein, die versorgt und gepflegt werden müssen? Dabei ist zu beachten, dass die Zahl der Menschen im mittleren Alter, die diese Pflege erwirtschaften und leisten können, stetig abnimmt. Für die Prognose des Pflegebedarfs insgesamt ist die Frage entscheidend, ob die Pflegewahrscheinlichkeit im höheren Lebensalter, z. B. durch medizinische Fortschritte, stark reduziert werden kann oder ob der Pflegebedarf gleich bleibt oder noch steigen wird, wenn die Zahl älterer Menschen in der Bevölkerung weiter zunimmt (vgl. z. B. Jacobzone et al. 2000; Hackmann \& Moog 2008). 
Zum Anderen stellt sich die Frage, wie sich der erwartete demografische Wandel auf den Arbeitsmarkt auswirken wird: Lassen sich gegenwärtige Muster der Erwerbstätigkeit beibehalten, wenn die Bevölkerung zunehmend altert? Und werden ältere Arbeitnehmer über ausreichendes Wissen und das notwendige Humankapital verfügen, um sich den Herausforderungen des Arbeitsmarktes anzupassen?

Für diese beiden Bereiche soll im Folgenden dargestellt werden, inwiefern Bildung als ein zentraler Faktor zur Bewältigung zukünftiger Herausforderungen beitragen kann. Dabei gilt es zu beachten, dass wir keinesfalls davon ausgehen, dass zukünftige demografische Herausforderungen allein durch Bildungspolitik zu bewältigen sind; vielfach bedarf sie - wie zu zeigen sein wird - einer Verzahnung mit anderen Politikfeldern, etwa der Arbeitsmarkt-, Familien- und der Sozialpolitik. Nichtsdestotrotz sind Investitionen in eine verantwortliche Bildungspolitik in Zeiten des demografischen Wandels unerlässlich, um zukünftigen Herausforderungen in angemessener Weise begegnen zu können.

\subsection{Gesundheit: Sozioökonomische Faktoren und Langlebigkeit}

Inwieweit eine wachsende Zahl an Älteren und Hochbetagten für eine Gesellschaft zur Bürde wird, hängt in starkem Maße davon ab, ob die steigende Lebenserwartung zugleich mit einem Anstieg der Lebenserwartung in einem guten Gesundheitszustand einhergeht. Es gibt Hinweise darauf, dass der Anstieg der Lebenserwartung bis ins sehr hohe Alter nicht zwangsläufig zu einem proportionalen Anstieg der Zahl Pflegebedürftiger führen muss (Doblhammer \& Ziegler 2006). Vieles spricht dafür, dass die erwartete steigende Lebenserwartung mit einer längeren Lebensphase ohne gesundheitliche Einschränkungen (disability-free life expectancy; DFLE) einhergehen könnte.

Doch an den Aussichten auf ein langes gesundes Leben partizipieren nicht alle gesellschaftlichen Gruppen gleichermaßen. Viele Studien haben gezeigt, dass sich soziale Benachteiligung auf die Chancen der Betroffenen auswirken kann, ein hohes Lebensalter zu erreichen (Valkonen 2006; Doblhammer et al. 2008). Die Diskrepanz nimmt dabei keineswegs ab: Neuere Studien, etwa für Österreich (Doblhammer et al. 2005; Schwarz 2005; Rau et al. 2008), aber auch für andere europäische und nordamerikanische Staaten (Mackenbach et al. 2003; Kunst et al. 2004; Valkonen 2006), weisen im Gegenteil auf wachsende sozioökonomische Disparitäten der Mortalität für Menschen im mittleren Alter bis 65 Jahre hin.

Dass Menschen mit niedrigem sozioökonomischem Status eine höhere Sterblichkeit haben als solche mit höherem Status, ist seit mehr als hundert Jahren bekannt. Dieses Verhältnis - je höher der soziale Status, desto niedriger die Sterblichkeit - wird unabhängig vom gewählten Indikator (ausgeübter Beruf, höchster Bildungsabschluss, Einkommen) gemessen. Insbesondere die Bildung ist ein guter Indikator, wie beispielsweise Valkonen (1989) darlegt: Bildung ist geringeren Fluktuationen ausgesetzt als andere Merkmale. Die relative Konstanz des Bildungsniveaus erleichtert nicht nur die empirische Erhebung und Analyse. Mit dem höchsten Bildungsabschluss lassen sich auch noch im Rentenalter soziale Unterschiede nachweisen, wenn die Trennschärfe anderer Indikatoren wie Beruf (,Rentner/Rentnerin“) abgenommen hat. Vielleicht von noch größerer Bedeutung ist die Tatsache, dass Bildung auch im Falle diskontinuierlicher Erwerbskarrieren für Frauen und Männer gleichermaßen über den Lebensverlauf hin gemessen werden kann. 
Der Einfluss der Bildung auf die Lebenserwartung in Gesundheit ist indirekter Natur, denn der Erwerb einer spezifischen Bildungsqualifikation impliziert nicht per se, dass ein Individuum höhere oder geringere Chancen hat, ein hohes Alter zu erreichen. Eine zentrale Rolle für die Langlebigkeit spielen vielmehr gesundheitsschädliche Lebensstile und Verhaltensweisen wie übermäßiger Alkoholkonsum, Rauchen oder schlechte Ernährung, die nachweislich durch Bildung beeinflusst werden (siehe z. B. Lynch et al. 1997) und ihrerseits zu wesentlichen Determinanten der Sterblichkeit zählen (Marmot 2005; Doblhammer et al. 2008). Es ist zu vermuten, dass die Variable „Bildung“ in Zukunft noch an Bedeutung gewinnen wird, da infolge des Wandels hin zu einer Dienstleistungsund Wissensgesellschaft die Relevanz berufsspezifischer Risiken für die Gesundheit und das Überleben wohl weiterhin zurückgehen wird.

Auf welche Todesursachen wirken sich nun diese durch Bildung gemessenen sozioökonomischen Unterschiede in der Sterblichkeit aus, die - insbesondere für Männer - über die Zeit hinweg noch zunehmen? Auch wenn es kein universales Muster gibt, so lassen sich doch einige weit verbreitete Trends beobachten (Koskinen 2003). Am Beispiel Österreichs zeigen Rau et al. (2008), dass bei Männern mit höherem sozioökonomischen Status die Sterblichkeit von Herz-Kreislauf-Erkrankungen überproportional gesenkt werden konnte. Die wachsenden Unterschiede über die Zeit hinweg liegen also nicht darin begründet, dass etwa die Sterblichkeit von Personen mit niedrigerem sozioökonomischen Status stabil bliebe oder gar zunähme. Die Mortalität sinkt in allen sozialen Gruppen, aber schneller für Männer mit höherem Bildungsabschluss. Bei der Betrachtung der Todesursache Herz-Kreislauf-Erkrankungen bei Frauen zeigt sich in Österreich auch hier ein Auseinanderdriften der Sterblichkeit zwischen Frauen mit hohem und niedrigem Bildungsstand. Allerdings gleichen andere zum Tode führende Krankheiten diesen Effekt bei Frauen aus. ${ }^{5}$

Sind es nur unterschiedliche Verhaltensweisen, die die (wachsenden) sozioökonomischen Diskrepanzen in der Sterblichkeit begründen? Oder lassen sich andere Faktoren anführen? In den vergangenen Jahrzehnten haben medizinische Fortschritte in der Prävention (z. B. Beta-Blocker) und der Behandlung (z. B. Bypass-Operationen, Herzschrittmacher) $\mathrm{zu}$ einem starken Rückgang in der Sterblichkeit aufgrund von HerzKreislauf-Erkrankungen geführt („cardiovascular revolution“, Vallin \& Meslé 2001). Es liegt die Vermutung nahe, dass Personen mit höherer Bildung auch tendenziell über ein höheres Einkommen verfügen und daher eventuell auch besseren Zugang zu neueren medizinischen Technologien besitzen. Für Österreich schließen Rau et al. (2008) diesen Mechanismus jedoch weitgehend aus, da in diesem Land ein nahezu egalitäres Gesundheitssystem besteht, zu dem alle Personen annähernd gleichen Zugang haben.

Bei der Betrachtung des Zusammenhangs zwischen Bildung und gesundheitsbewusstem Verhalten muss jedoch beachtet werden, dass spezifische Verhaltensweisen, die sich negativ auf die Gesundheit und die Lebenserwartung auswirken, für bestimmte Alterskohorten prägend sein können, bisweilen sogar Teil einer bestimmten Sozialisierung sind. So können etwa Fortschritte im Anstieg der Lebenserwartung, die im Laufe der Jahre erreicht werden, durch kohortenspezifische Rückschritte wieder aufgewogen werden. Diese kohortenspezifischen Unterschiede im Gesundheitsverhalten könnten einen der allgemein steigenden Lebenserwartung entgegenstehenden Einfluss auf krankheitsspezifische Sterberaten haben. ${ }^{6}$ 
Trotz dieser kohortenspezifischen Effekte wurde jedoch immer wieder bestätigt, dass Bildung einen positiven Einfluss auf die Lebenserwartung hat. Es liegt auf der Hand, dass Bildung somit auch die Lebenserwartung in Gesundheit beeinflusst. So zeigt eine Studie, die Unterschiede in gesunden Lebensjahren (healthy life years, HLYs) in 25 EUMitgliedsstaaten erfasst, dass für Männer mit höherer Bildung im Alter von 50 Jahren die zu erwartende verbleibende Zahl an gesunden Lebensjahren größer ist als die für Männer gleichen Alters mit niedrigerer Bildung (Jagger et al. 2008).

Die durchschnittlich zu erwartende Zahl an gesunden Lebensjahren ist für eine Gesellschaft eine entscheidende Größe, da von ihr die künftigen Belastungen der Gesundheitsund Pflegesysteme sowie des Rentensystems abhängen. In Zeiten, da angesichts einer alternden Gesellschaft über eine Verlängerung der Lebensarbeitszeit diskutiert wird, ist der Gesundheitszustand der sogenannten ,jungen Alten“ zwischen 60 und 80 Jahren ein ausschlaggebender Faktor dafür, ob das Erwerbspotenzial älterer Menschen auch tatsächlich genutzt werden kann. Die Studie von Jagger et al. (2008) zeigt auch, dass die Differenz in der Anzahl der gesunden Lebensjahre, die im Alter von 50 Jahren zu erwarten ist, zwischen den europäischen Ländern deutlich größer ist als die Länderunterschiede in der Restlebenserwartung mit 50 Jahren. Besonders niedrige Werte für die erwarteten gesunden Altersjahre finden sich in einigen neuen EU-Mitgliedstaaten. Doch auch deutsche Männer und Frauen zeigten nach dieser Studie im Jahr 2005 mit jeweils 13,6 Jahren eine im europäischen Vergleich geringe Zahl an zu erwartenden gesunden Lebensjahren (durchschnittliche HLYs in 25 untersuchten EU-Staaten: Männer 17,3 und Frauen 18,1 Jahre). Die Entwicklung in Deutschland kann als problematisch bezeichnet werden, da die gesamte Restlebenserwartung im Alter von 50 Jahren (Männer 29 Jahre, Frauen 33,4 Jahre) deutlich über der Zahl der zu erwartenden gesunden Lebensjahre liegt. Diese Entwicklung kann ihrerseits Implikationen für die Möglichkeiten der Verlängerung der Lebensarbeitzeit und der Beschäftigungsquoten älterer Arbeitnehmer (vgl. Kapitel 2.2) haben. Hier besteht daher ein dringender politischer Handlungsbedarf.

Zusammenfassend lässt sich somit festhalten, dass der Bildung bei der Sicherstellung einer weitgehend beschwerdefreien Altersphase eine zentrale Rolle zukommt. Dennoch bedarf es angesichts des bislang begrenzten Datenmaterials und Erkenntnisstandes weiterer Forschungen, vorzugsweise im Längsschnittdesign, um die Determinanten der Gesundheit im hohen Alter besser zu verstehen (Richter \& Hurrelmann 2006; Hradil 2006).

\subsection{Die Umverteilung von Arbeit im Lebenslauf}

Neben den gesundheitlichen Konsequenzen hat die steigende Wahrscheinlichkeit, ein hohes Alter zu erreichen, Auswirkungen auf die Gestaltung des Lebenslaufs und die Balance von Lebens- und Arbeitszeit. Die starre Dreiteilung klassischer Erwerbsbiografien, die das Lernen in der ersten Lebensphase, das Arbeiten in der Mitte des Lebens und die Freizeit in den späten Jahren konzentriert, löst sich zunehmend auf (vgl. z. B. Heinz 2003; Marshall \& Müller 2002; Naegele et al. 2003). Das dreiteilige Lebenslaufmodell gerät zurzeit in mehrfacher Hinsicht unter Druck: 
- Gegenwärtig sind in Deutschland die Ausbildungszeiten lang und der Weg in das Erwerbsleben ist bisweilen durch längere Wartezeiten sowie ein hohes Maß an Unsicherheit bis zum Erreichen einer stabilen Beschäftigung gekennzeichnet. In Zeiten zunehmender Globalisierung und Flexibilisierung erweisen sich dabei insbesondere junge Menschen mit geringen bzw. nur basalen Bildungsabschlüssen als ,Verlierer des sozialen Wandels (vgl. Blossfeld et al. 2005, 2009). In Deutschland nimmt der Umfang dieser Gruppe von ,Bildungsverlieren“ gegenwärtig zu. Grundlegend hierfür ist, dass aus der „Bildungsexpansion“ der 1970er-Jahre zunehmend eine „Bildungsstagnation“"wurde (Allmendinger \& Ebner 2006). Zum Einen erreicht in Deutschland nur ein vergleichsweise geringer Anteil der Bevölkerung einen Abschluss im Tertiärbereich (OECD 2008, S. 44). Zum Anderen befindet sich eine relativ hohe Anzahl an Schulabsolventen in sogenannten „Übergangsmaßnahmen“, d.h. in Bildungsangeboten unterhalb des Status einer festen Berufsausbildung, die primär auf den Erwerb persönlicher Kompetenzen sowie das Nachholen eines schulischen Abschlusses fokussieren (Autorengruppe Bildungsberichterstattung 2008). Vielen Jugendlichen gelingt es nur bedingt, diese Systeme in Richtung einer allgemeinen beruflichen Qualifizierung zu verlassen (ebd; Baethge et al. 2007). Sie verbleiben somit dauerhaft ohne einen beruflich qualifizierenden Abschluss. Derartig prekäre Bildungskarrieren haben negative Konsequenzen sowohl für das persönliche Leben als auch für die Gesellschaft, geht dieser langfristig doch qualifiziertes Arbeitskräftepotenzial verloren. Der vielfach beklagte „Fachkräftemangel“" wird dadurch noch verschärft (Reinberg \& Hummel 2004).

- Die Entwicklungsmuster der Arbeitsmarktsituation junger Menschen bleiben zudem nicht ohne Auswirkungen auf die Familien- und Fertilitätsentwicklung sowie damit auf die langfristige Entwicklung der Bevölkerungsstruktur. Blossfeld et al. (2005) verweisen darauf, dass verlängerte Ausbildungszeiten oftmals als „Warteschleife“ bzw. zum Erwerb weiterer Qualifikationen vor dem oftmals schwierigen Eintritt in rigide Arbeitsmarktstrukturen genutzt werden. Durch diesen verzögerten Eintritt in das eigentliche Arbeitsleben kommt es jedoch auch zum Aufschieben von Geburten, da die Familiengründung meist erst nach der erfolgreichen Etablierung auf dem Arbeitsmarkt erfolgt. Frejka und Sobotka (2008) sehen in diesem Aufschub der Familiengründung und der Geburt des ersten Kindes einen wesentlichen Faktor für das Absinken der Geburtenraten in Europa. Während es in einigen Ländern, etwa in Frankreich, zu einem fast vollständigen ,Aufholen“ dieses Aufschubs gegen Ende der reproduktiven Phase kommt, so dass dort die Zahl der Zweit- und Drittgeburten zunimmt, hat ein derartiger Aufholprozess in Deutschland und in vielen anderen Ländern Europas nicht stattgefunden.

- Problematisch stellt sich auch die Situation von Frauen auf dem deutschen Arbeitsmarkt dar. Im Zuge der Bildungsexpansion ist in den vergangenen Jahren ihr Anteil an den höheren Segmenten des Bildungssystems deutlich gestiegen. In der tertiären Bildung haben junge Frauen ihre männlichen Altersgenossen bereits überholt (vbw 2009). Gleichwohl schlägt sich dieser Zuwachs bislang kaum in einer entsprechenden Arbeitsmarktbeteiligung von Frauen nieder. Zwar sind heutzutage mehr als zwei Drittel aller deutschen Frauen im erwerbsfähigen Alter auch de facto erwerbstätig. Insbesondere Frauen mittleren Alters, also zwischen 30 und 50 Jahren, stehen jedoch durch 
die gleichzeitigen Anforderungen des Familienlebens und der Erwerbstätigkeit in einem sich verändernden Arbeitsmarkt oftmals unter einer starken Belastung (Blossfeld \& Hofmeister 2006). Ein großer Teil deutscher Frauen unterbricht daher im Falle einer Familiengründung die Erwerbstätigkeit und kehrt anschließend meist lediglich in Teilzeitarbeit wieder in den Arbeitsmarkt zurück (Blossfeld et al. 2001). Auch hierdurch geht dem deutschen Arbeitsmarkt in Zeiten des demografischen Wandels ein großes Potenzial an oftmals gut qualifizierten Arbeitskräften verloren. Bislang gibt es kaum Anzeichen dafür, dass sich dieses geschlechtsspezifische Verhalten am Arbeitsmarkt ändert, da es an kostengünstigen Möglichkeiten zur frühkindlichen Betreuung mangelt und Männer im Durchschnitt höhere Einkommenschancen haben, insbesondere in typischen Männerberufen. Durch jüngere sozioökonomische Entwicklungen hat sich die Situation von Frauen am Arbeitsmarkt zudem kaum verbessert. So zeigen etwa Buchholz und Grunow (2006), dass die Erwerbskarrieren von deutschen Frauen durch die Globalisierung zunehmend unsicherer geworden sind. Im Übrigen müssen viele Frauen angesichts sinkender Rentenerwartungen verstärkt für ihr eigenes Alter vorsorgen (Allmendinger 2000), was ihre Situation umso prekärer macht.

- Schließlich erfolgt der Ausstieg aus dem Erwerbsleben in Deutschland bislang immer noch sehr früh. Unter den 60- bis 64-Jährigen ist nach einem nahezu kontinuierlichen Rückgang der Erwerbstätigkeit in diesem Alterssegment seit den 1970er-Jahren in Deutschland heute etwa nur noch ein Drittel überhaupt erwerbstätig (OECD 2007); es folgen Jahrzehnte des sogenannten „Ruhestands“. Diese Entwicklung hat in vielen europäischen Staaten dazu beigetragen, dass sich die Rentensysteme einer ,zweifachen Finanzierungskrise" gegenüber sehen: Einerseits verursachen geringe Fertilitätsraten langfristig eine Verringerung der Anzahl der Beitrags- und Steuerzahler zur Sozialversicherung; andererseits hat der immer frühere Austritt aus dem Erwerbsleben eine Erhöhung der Bezieher von Rentenleistungen oder anderen Sozialtransfers zu Folge (etwa der Arbeitslosen- oder der Invalidenversicherung, die vielfach als „Brücke in den Ruhestand“ genutzt werden; vgl. Blossfeld et al. 2006; Buchholz 2008; Hofäcker, in press; Hofäcker et al. 2007). Der bevorstehende Renteneintritt der geburtenstarken „Babyboomer“ wird dieses wachsende Ungleichgewicht zwischen Beitragszahlern und Leistungsempfängern weiter verstärken. Im Hinblick auf diese Entwicklungen stand Deutschland 2005 im europäischen Vergleich zwar nicht allein da. Gleichwohl gibt es Länder mit deutlich höheren Beschäftigungsquoten von Älteren, insbesondere in den skandinavischen Staaten sowie im angelsächsischen Sprachraum. In dem Maße, in dem die Älteren auf dem deutschen Arbeitsmarkt nicht berücksichtigt werden, bleibt ein weiteres erhebliches Arbeitspotenzial ungenutzt. Wenn hier keine Änderung eintritt, könnte dies bedeuten, dass in Zukunft noch mehr Menschen als heute nicht am Erwerbsleben beteiligt sind. Die Arbeit wäre dann noch ungleichmäßiger verteilt und die Last für die Jüngeren, die Älteren über die Sozialsysteme zu unterstützen, könnte sich dadurch weiter vergrößern.

Vor dem Hintergrund der verlängerten Lebensläufe und der wachsenden Zahl älterer Erwerbsfähiger in den kommenden zwei Jahrzehnten erscheint ein Festhalten an dem bisherigen Lebenslaufmuster obsolet. Angesichts des demografischen Wandels scheint es vielmehr geboten zu sein, die starre Dreiteilung des Lebenslaufs aufzugeben, die 
Beschränkung der Ausbildungsphase allein auf das frühe Lebensalter aufzulösen und die verdichtete Erwerbsphase zwischen 30 und 50 Jahren zu entzerren.

Die ökonomischen Konsequenzen der demografischen Umbrüche können mit dem am Rostocker Zentrum zur Erforschung des demografischen Wandels entwickelten Rostocker Indikator (Vaupel \& Loichinger 2006) genauer quantifiziert werden. Der Indikator beschreibt, wie schnell sich die insgesamt geleistete Arbeitszeit in einer alternden Gesellschaft verringern würde, wenn sich nichts an der gegenwärtigen Verteilung der Lebensarbeitszeit ändert. Der Rostocker Indikator basiert auf der durchschnittlich gearbeiteten Stundenzahl pro Kopf und Woche in der Bevölkerung - vom Säugling bis zum Greis, gleich ob erwerbstätig oder nicht. Für das Jahr 2005 betrug er in Deutschland 16,3 Stunden. ${ }^{7}$ Durch den demografischen Wandel ändert er sich jedoch im Laufe der Zeit. Lag er vor 20 Jahren in Deutschland aufgrund einer höheren Beschäftigtenquote noch bei 16,9 Stunden pro Kopf und Woche, so wird er bis 2025 bei gleichbleibenden altersspezifischen Beschäftigungsquoten um 8\% auf 15 Stunden sinken. Die Berechnungen zeigen, dass die kollektive Alterung bereits innerhalb kurzer Zeit zu wirtschaftlichen Einbußen in Deutschland führen könnte, sofern sich die altersspezifischen Beschäftigungsquoten nicht ändern.

Wenn dagegen die Arbeitsleistung gleichmäßiger über den Lebenslauf von Männern und Frauen verteilt würde, ließe sich die durchschnittliche Lebensarbeitszeit deutlich erhöhen. Hierzu bieten sich angesichts der gegenwärtigen geschlechtsspezifischen Struktur der Erwerbstätigkeit im Lebenslauf verschiedene Möglichkeiten: Es könnte beispielsweise eine Erhöhung der bisherigen Erwerbsquote von Müttern, und dabei insbesondere eine Erhöhung ihres durchschnittlichen Arbeitsvolumens, angestrebt werden (Allmendinger \& Ebner 2006; Allmendinger et al. 2006). Eine zentrale Bedeutung kommt in diesem Zusammenhang Maßnahmen zur besseren Vereinbarkeit von Familie und Beruf zu. Diese würden es nicht nur Frauen mit Kindern erleichtern, ihre Erwerbstätigkeitsphase zu verlängern und den Umfang ihrer Erwerbstätigkeit zu erhöhen. Gleichzeitig würden sie dazu beitragen, dass Familiengründung und Berufsorientierung nicht mehr zwangsläufig in einem Konkurrenzverhältnis zueinander stehen. Hierdurch ließe sich möglicherweise die Bereitschaft zur Familiengründung potenziell erhöhen, die Fertilität steigern und damit auch langfristig die demografische Entwicklung beeinflussen. Begleitet werden müssten derartige Maßnahmen durch eine Angleichung der geschlechtsspezifischen Einkommenschancen, um die gegenwärtig zu beobachtende Spezialisierung von Männern und Frauen auf verschiedene Lebensbereiche aufzubrechen. Hierzu gehören etwa eine Verringerung der ungleichen Verteilung der Geschlechter auf verschiedene Berufsfelder (Allmendinger et al. 2006). Die Bildungspolitik könnte hierzu durch die Förderung einer eher geschlechterneutralen Berufswahl ebenso wie durch die Förderung von Fort- und Weiterbildung für Frauen im Falle eines vorübergehenden Erwerbsausstiegs beitragen (vbw 2009).

Die gegenwärtige demografische Entwicklung zeigt jedoch, dass zur Aufrechterhaltung eines angemessenen Arbeitskräfteangebots neben der Förderung der Erwerbstätigkeit von Frauen insbesondere die Erwerbsbeteiligung Älterer erhöht werden muss. In der Bevölkerungspyramide von heute bilden die sogenannten „Babyboomer“ zurzeit eine weite Ausbuchtung in der Mitte der Pyramide (vgl. Abb. 2). Sie stehen in großer Zahl für das Erwerbsleben bereit und haben wesentlich weniger Kinder zu versorgen als vorangegangene Generationen. Im Hinblick auf die verfügbare Arbeitskraft sind das besonders 


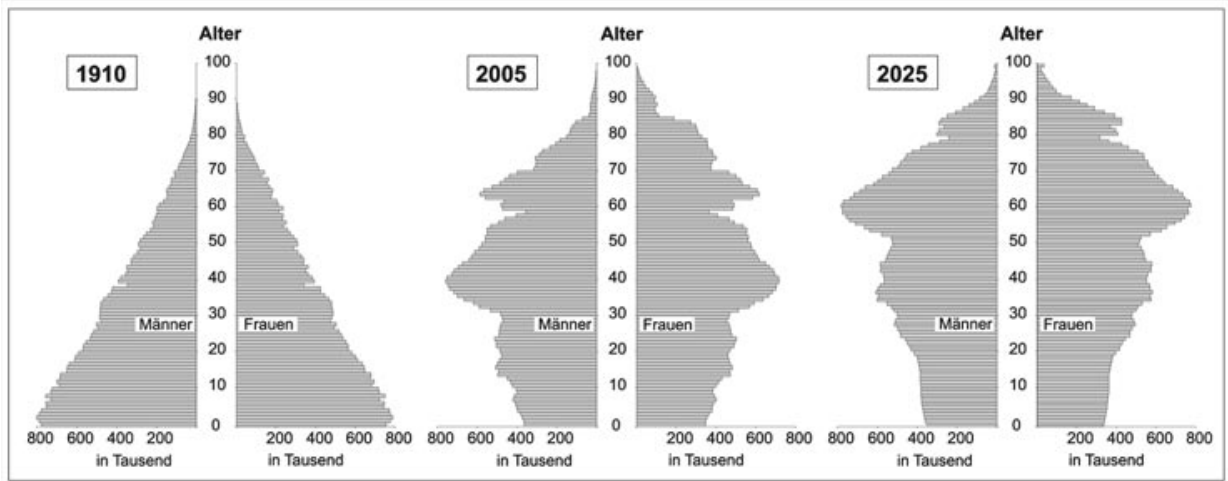

Abb. 2: Bevölkerungspyramiden für Deutschland in den Jahren 1910, 2005 und vorausberechnet für das Jahr 2025 (nach der 11. Koordinierten Bevölkerungsvorausberechnung des Statistischen Bundesamtes, Wiesbaden, Variante 2-W1). Die Bevölkerungsstruktur von 1910 ergibt noch eine Pyramidenform mit einer breiten Basis von vielen Kindern und einer schmaler werdenden Spitze, geformt durch wenige Alte. Im Jahr 2005 zeigt sich eine Ausbuchtung um die Mitte, gebildet von den geburtenstarken Jahrgängen. Diese Ausbuchtung rückt Jahr für Jahr weiter auf und erreicht bis 2025 das Alter 60 Jahre. Adaptiert nach Vaupel \& Loichinger 2006.

günstige Bedingungen. Die Ältesten unter ihnen sind heute Mitte 50 und in etwa 15 Jahren wird die Mehrheit der geburtenstarken Jahrgänge etwa 60 Jahre alt sein. Unterhalb der Ausbuchtung verjüngt sich das, was einmal die Basis einer echten Pyramidenform war, merklich und es kommt zu der häufig als „Urnenform“ bezeichneten Darstellung des Bevölkerungsaufbaus. Entsprechend niedriger ist in jedem Jahrgang die Zahl der jungen Menschen; ihr Anteil wird zukünftig weiter deutlich sinken. ${ }^{8}$

Die Frage, in welchem Maß die Erwerbsfähigen insgesamt und auch die über 50-Jährigen ihre Arbeitskraft einsetzen, ist somit für die ökonomische Zukunft Deutschlands von großer Bedeutung (Börsch-Supan et al. 2005). Die Situation älterer Arbeitnehmer auf dem deutschen Arbeitsmarkt ist nach wie vor kritisch, trotz des Anstiegs ihrer Beschäftigungsquoten in der jüngeren Vergangenheit. Ihre Erwerbsquote im „Vorruhestandsalter“ von 60 bis 64 Jahren liegt weiterhin deutlich unter 50 Prozent. Zudem befindet sich ein hoher Anteil älterer Arbeitnehmer gegenwärtig in - meist langfristiger - Altersarbeitslosigkeit (vgl. Buchholz 2008; Hofäcker in press).

Diese problematische Situation älterer Arbeitnehmer lässt sich dabei nicht auf gesundheitliche Einschränkungen zurückführen. Zwar stellen sich die Arbeitsmöglichkeiten über 60-Jähriger je nach Gesundheitszustand und Belastung durch den ausgeübten Beruf unterschiedlich dar. Jedoch gilt es zu beachten, dass Menschen in ihren 60ern heute im Durchschnitt ,jünger" und leistungsfähiger sind als noch vor einigen Jahrzehnten (Crimmins et al. 1999). Die moderne Arbeitswelt fordert immer weniger starke körperliche Arbeit. Gerade der wachsende Dienstleistungssektor könnte - bei adäquater Qualifikation die Älteren der Zukunft als Arbeitskräfte aufnehmen.

Die Benachteiligungen älterer Arbeitnehmer am Arbeitsmarkt lassen sich ebenso wenig auf ihre vermeintlich geringere Produktivität zurückführen (vgl. z. B. Auer \& Fortuny 2000; Kohli 1985). ${ }^{9}$ Ältere verfügen über wertvolles Erfahrungswissen - auch wenn andere Fähigkeiten, die etwa physische Stärke erfordern, zurückgehen mögen. Ältere 
Arbeitnehmer in Deutschland werden de facto allerdings - trotz einer teilweise durchaus positiven Einschätzung ihrer Fähigkeiten - von Unternehmen nur selten gefördert oder neu eingestellt. Die Wahrnehmung Älterer als „Schwachstelle“ im Betrieb ist oftmals nicht nur ungerechtfertigt, sondern verhindert vielfach auch, dass Arbeitgeber in die Weiterqualifikation ihrer älteren Belegschaft investieren.

Wesentliche Gründe aus Unternehmenssicht stellen hierbei die bei älteren Mitarbeitern vergleichsweise hohen „Senioritätslöhne“, ein oft besserer Kündigungsschutz sowie in Zeiten rapiden technologischen Wandels vor allem auch veraltete Qualifikationen dar, die im Lebensverlauf nur selten ,,aufgefrischt“ wurden (Hofäcker et al. 2007; Schröder et al. 2009). Aktuelle Studien zeigen, dass die Wiedereinstellungschancen älterer Arbeitsuchender nach wie vor meist schlechter sind als bei jüngeren Arbeitsmarktkonkurrenten (Frosch 2007), die über einen geringeren Kündigungsschutz verfügen, deren Löhne und Arbeitsverhältnisse sich leichter flexibilisieren lassen und deren Qualifikationen eher den Anforderungen der modernen Dienstleistungsgesellschaft entsprechen. In den vergangenen Jahrzehnten wurden daher für ältere Arbeitnehmer durch einen Ausbau gesetzlicher Frühverrentungsmöglichkeiten bzw. weiterer Pfade in den vorzeitigen Ruhestand starke finanzielle Anreize gesetzt, frühzeitig aus dem Erwerbsleben auszuscheiden (vgl. Arnds \& Bonin 2003; Buchholz 2008).

Angesichts der demografischen Entwicklung sind jedoch eine Umkehr des Frühverrentungstrends sowie eine deutliche Erhöhung der Erwerbstätigkeit älterer Arbeitnehmer mittelfristig unumgänglich. Im Jahr 2005 arbeiteten 45-Jährige durchschnittlich 30 Stunden pro Kopf und Woche, 60-Jährige dagegen nur acht. Soll die Zahl der durchschnittlich in Deutschland geleisteten Wochenarbeitsstunden nicht deutlich sinken, müsste sich dieses Muster deutlich ändern. In den Modellrechnungen wäre dies in unterschiedlicher Form vorstellbar: So müsste etwa bis 2025 erreicht werden, dass die 50- bis 60-Jährigen wie die 35- bis 50-Jährigen etwa 30 Stunden arbeiten und dass die Arbeitszeit der 60- bis 65-Jährigen auf 20 Stunden steigt (vgl. Abb. 3). Dann bliebe der wirtschaftliche Arbeitseinsatz auch bei einer alternden Erwerbsbevölkerung konstant. Würde bis zum Alter von 65 Jahren auf gleichbleibend hohem Zeitniveau gearbeitet und wären Menschen bis zum Alter von 70 Jahren zu einem gewissen Teil in das Erwerbsleben eingebunden, ließe sich sogar eine Entlastung für jüngere Altersgruppen bewerkstelligen, etwa für junge Erwerbstätige zwischen 20 und 40 Jahren.

Vor diesem Hintergrund gibt es gute Argumente dafür, die heute immer noch gängige Personalpolitik zu ändern, die Arbeitnehmern im Alter von über 50 Jahren kaum Chancen bietet. Es existiert ein solides Fundament an demografischen und ökonomischen Studien, um politische Entscheidungsträger und die Öffentlichkeit über die Optionen zu informieren, die mit der Steigerung der Beschäftigungsquote älterer Arbeitnehmer verbunden sind (OECD 2001; Reday-Mulvey 2005).

Es ist dabei insbesondere darauf hingewiesen worden, welche zentrale Rolle Aus- und Weiterbildungsmaßnahmen spielen können, um in Zeiten einer zunehmend alternden und schon in wenigen Jahren schrumpfenden Erwerbsbevölkerung das vorhandene Erwerbspotenzial zu nutzen (Börsch-Supan 2003) und dem Einzelnen Entwicklungsmöglichkeiten im Laufe seiner Erwerbsbiografie zu eröffnen.

Aus betrieblicher Sicht stellen Qualifikationsdefizite älterer Arbeitnehmer in Deutschland gegenwärtig einen wesentlichen Aspekt ihrer Wettbewerbsnachteile gegenüber jün- 


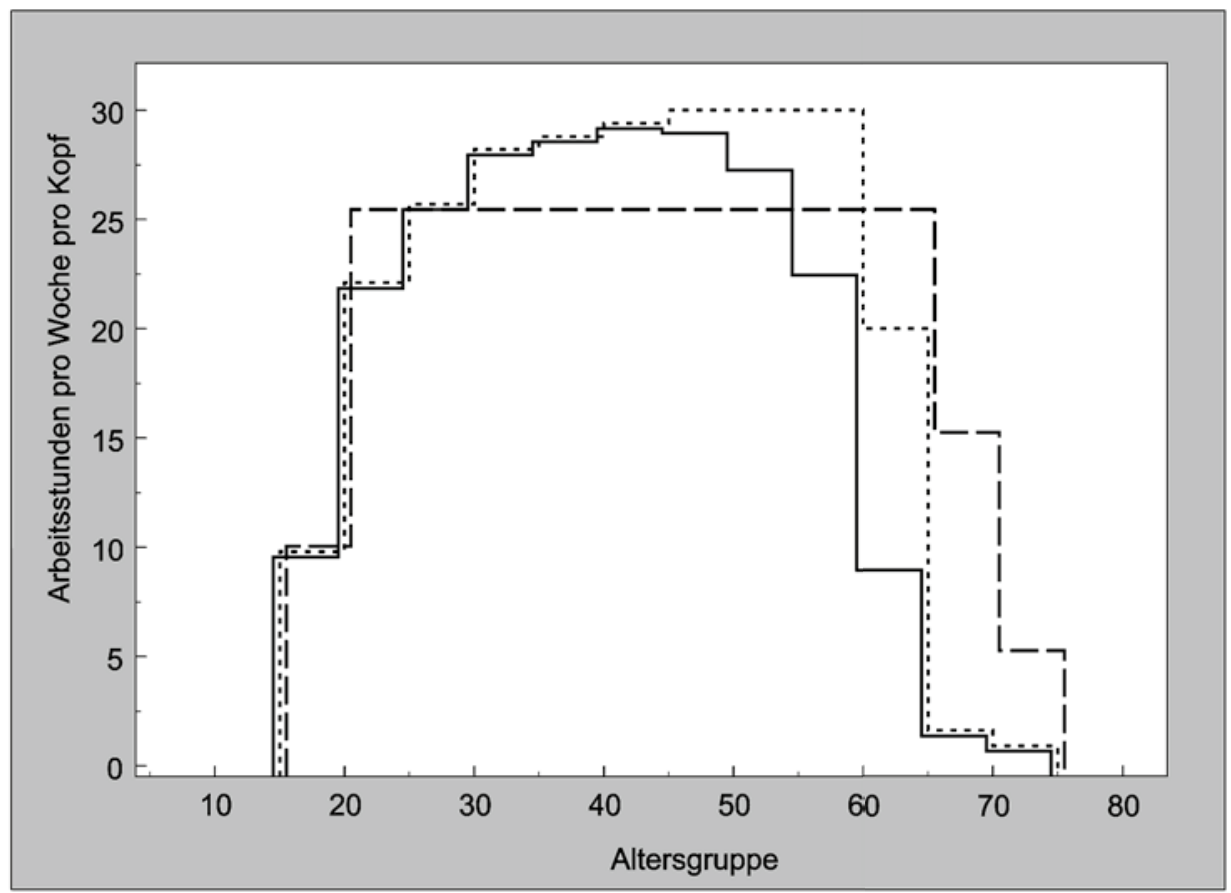

\begin{abstract}
Abb. 3: Durchschnittliche Zahl der pro Woche und Kopf gearbeiteten Stunden in Deutschland nach Altersgruppen. Die durchgezogene Linie beschreibt das Muster in 2005, das insgesamt zu einer durchschnittlichen Wochenarbeitsstundenzahl von 16,3 führt. Die fein gestrichelte Linie zeigt als Modellrechnung, dass ein gesteigerter Arbeitseinsatz von älteren Beschäftigten 2025 die durchschnittlich geleistete Wochenarbeitsstundenzahl auch bei veränderter Bevölkerungsstruktur konstant halten könnte. Die lang gestrichelte Linie illustriert eine Möglichkeit, die Arbeit noch gleichmäßiger über die Altersgruppen zu verteilen, ohne Einbußen in der insgesamt geleisteten Arbeitszeit zu bewirken: Menschen zwischen 20 und 65 Jahren würden durchschnittlich 25,1 Stunden pro Woche arbeiten. Quelle: Vaupel \& Loichinger 2006.
\end{abstract}

geren Arbeitnehmern dar (Hofäcker et al. 2008). Wie bereits dargestellt, werden diese angesichts mangelnder Auffrischung im Lebensverlauf vielfach als veraltet angesehen; betriebliche Investitionen in eine Weiterqualifizierung älterer Arbeitskräfte erscheinen angesichts der geringen verbleibenden Restarbeitszeit zudem oftmals als nicht lohnend, wenn jüngere Arbeitskräfte mit aktuelleren Qualifikationen zur Verfügung stehen. Um die Wettbewerbsnachteile älterer Arbeitnehmer zu reduzieren, muss deren Fort- und Weiterbildung somit bereits vor der eigentlichen Altersphase einsetzen. Durch lebenslanges Lernen muss verhindert werden, dass sich über den Lebenslauf hinweg generationenspezifische Differenzen in Qualifikation und Kenntnissen herausbilden. Jüngere Studien belegen in der Tat, dass Länder, die aktiv in die Förderung lebenslangen Lernens investieren (v. a. die skandinavischen Staaten) bzw. in denen kontinuierliches ,Lernen am Arbeitsplatz" eine zentrale Rolle spielt (v. a. die angelsächsischen Länder), eher in der Lage sind, ältere Arbeitnehmer im Erwerbsleben zu halten, als beispielsweise Deutschland (vbw 2008; Ebbinghaus 2005; Hofäcker \& Pollnerová 2006). Regelmäßige „Aktualisierungen“ der Kenntnisse und Qualifikationen erleichtern es älteren Mitarbeitern in diesen Ländern, 
sich dauerhaft an den rasanten technologischen und arbeitsmarktstrukturellen Wandel der vergangenen zwei bis drei Jahrzehnte anzupassen und daher auch im höheren Alter im Vergleich zu jüngeren Arbeitnehmern ,wettbewerbsfähig“ zu bleiben.

Auch hier stellen die genannten bildungspolitischen Maßnahmen nur einen Teilaspekt eines umfassenderen politischen Handlungsfeldes dar. Begleitet werden müssen sie durch weitere Maßnahmen, die einer Herausbildung bzw. Verfestigung von Arbeitsmarktnachteilen älterer Arbeitnehmer entgegenwirken, etwa einer aktiven Arbeitsmarktpolitik oder einer Verringerung der (Lohn-)Kostennachteile älterer Arbeitnehmer. Der Investition in bildungspolitische Maßnahmen kommt ebenfalls wiederum eine zentrale Stellung zu, da diese letztlich durch die lebenslange Vermittlung beruflich relevanter Qualifikationen die Grundvoraussetzung für eine Weiterbeschäftigung älterer Arbeitnehmer schaffen.

Bereits seit langem wird darauf hingewiesen, dass Bildung das wesentliche Fundament bildet, auf dem die Menschen ihr langes Leben bei wechselnden Möglichkeiten und Bedingungen mit Sinn erfüllen und ein gutes Auskommen für sich und ihre Familien sichern können (Vaupel \& Owen 1986). In diesem Sinne ist nicht nur eine rein chronologische Verlängerung der Erwerbsbiografie entscheidend, sondern ebenso ihre aktive und kreative Gestaltung. Dies beinhaltet explizit die Notwendigkeit zu lebenslangem Lernen. Dazu gehören neue Möglichkeiten, die Lebensarbeitszeit eigenverantwortlich aufzuteilen und Lebensphasen für Arbeit, Familie, Bildung und gemeinnütziges Engagement wesentlich flexibler zu gestalten, als es heute üblicherweise der Fall ist (Vaupel \& Loichinger 2006; Vaupel \& Kistowski 2007). Eine derartige Herangehensweise erfordert eine verantwortungsvolle Planung der verschiedenen Lebensphasen, Eigeninitiative und die Entwicklung und Ergänzung von erforderlichen Fähigkeiten und Wissensinhalten. Nur so kann der Einzelne sich den veränderten Lebenssituationen und den von außen einwirkenden Bedingungen anpassen. Voraussetzung dafür ist, dass Bildungsangebote nicht erst im höheren Lebensalter einsetzen, sondern während des gesamten Lebens genutzt werden können. Bildungsangebote dürfen also nicht nur - gewissermaßen als „kompensatorische Maßnahme“ - für ältere Menschen zur Verfügung stehen. Vielmehr bedarf es einer kontinuierlichen Auffrischung von Kenntnissen und Wissensinhalten über den gesamten Lebensverlauf. In Deutschland zeigt sich diesbezüglich noch eine starke Beschränkung der beruflichen Bildung und Ausbildung auf die frühen Lebensjahre (vbw 2008). Im weiteren Berufsverlauf folgen nur selten bzw. nur für spezifische Arbeitnehmergruppen Aktualisierungen des zuvor erworbenen Wissens (OECD 1999). Um den Herausforderungen der Zukunft gewachsen zu sein, muss dem lebenslangen Lernen, d. h. der Ausweitung von Bildungsangeboten auch für ältere Menschen, eine größere Bedeutung eingeräumt werden.

\section{Die Rolle der Bildung bei den Ursachen und der Bewältigung des demografischen Wandels}

Bildung spielt sowohl bei der Betrachtung der Ursachen als auch hinsichtlich der Konsequenzen des demografischen Wandels eine wichtige Rolle. Im Vorangegangenen konnte dies etwa hinsichtlich der Verbesserung der Gesundheit, bei der Umverteilung von Arbeit 
oder bei der aktiven Gestaltung einer sich verlängernden Phase des Alterns gezeigt werden.

Darauf aufbauend, lassen sich einige Schlussfolgerungen für die Gestaltung zukünftiger Bildungspolitik sowie für die weitere Forschung ziehen:

1. Bildung darf sich nicht auf eine spezifische Lebensphase beschränken, sondern muss kontinuierlich und lebenslang erfolgen. Nur so lassen sich langfristig gleiche Arbeitsmarktchancen von Arbeitnehmern aus unterschiedlichen Altersgruppen sicherstellen und Wettbewerbsnachteile älterer Arbeitnehmer verringern. Eine kontinuierliche Bildung während des gesamten Lebenslaufs erhöht zudem die Chancen, die letzte Lebensphase gesund und selbstbestimmt verbringen zu können.

2. Männern und Frauen müssen langfristig gleiche Bildungs- und Arbeitsmarktchancen eingeräumt werden. Gegenwärtig befördern eine selektive Berufswahl von Männer und Frauen sowie daraus resultierende ungleiche Verdienstchancen am Arbeitsmarkt eine Spezialisierung von Geschlechterrollen nach traditionellem Vorbild. Hierdurch geht der deutschen Gesellschaft gegenwärtig ein beachtliches Arbeitskräftepotenzial verloren. Vor dem Hintergrund der zu erwartenden Alterung der Bevölkerung sollte eine derartig einseitige geschlechtsspezifische Arbeitsmarktteilnahme nicht hingenommen werden. Die Bildungspolitik muss künftig sicherstellen, dass Frauen und Männern ein gleicher Zugang zu ähnlichen Berufsfeldern ermöglicht wird und dass Geschlechterstereotypen in der Berufswahl nicht weiterhin zur Segregation im Arbeitsmarkt beitragen.

3. Angesichts der niedrigen Geburtenraten in Europa wurde vor dem Hintergrund einer wachsenden Zahl von Frauen mit einem höheren Bildungsabschluss die Frage aufgeworfen, ob es einen Zusammenhang zwischen dem Bildungsabschluss und Kinderlosigkeit gibt. Obwohl etwa in Deutschland eine höhere Kinderlosigkeit unter Akademikerinnen als unter Frauen mit niedrigeren Bildungsabschlüssen beobachtet werden konnte (Duschek \& Wirth 2005), ist der Zusammenhang zwischen Bildungsstand und Kinderlosigkeit keineswegs eindeutig. Während etwa in Schweden die Ausbildungsrichtung für das Ausmaß der Kinderlosigkeit entscheidender ist als die Bildungshöhe (Hoem et al. 2006), spielt in Österreich auch die Bildungshöhe eine entscheidende Rolle, was vor allem institutionellen Faktoren zugeschrieben wird (Neyer \& Hoem 2008). Diese national unterschiedlichen Ergebnisse verweisen auf die Bedeutung länderspezifischer Rahmenbedingungen für individuelle Entscheidungen an der Schnittstelle von Bildungsbiografien und Lebenslaufentscheidungen. Wichtig ist in diesem Zusammenhang, dass der Erwerb von Bildungsqualifikationen nicht in - zeitliche oder finanzielle - Konkurrenz zu individuellen Fertilitätsentscheidungen geraten darf, etwa dadurch, dass die individuelle Familiengründung mit einer unfreiwilligen vorübergehenden „Erwerbs-Auszeit“ einhergeht, in der der Zugang zu Bildung und zum Arbeitsmarkt verloren geht und damit die individuelle Erwerbskarriere beeinträchtigt wird. Bildungspolitik stellt hier entsprechend kein isoliertes Politikfeld dar, sondern steht in engem Zusammenhang mit arbeitsmarkt- und familienpolitischen Weichenstellungen.

4. Bildung muss allen gesellschaftlichen Gruppen in vergleichbarer Weise zugänglich sein. Gegenwärtig weist das deutsche Bildungssystem deutliche Tendenzen zu einer 
Polarisierung seiner Bildungsabgänger sowie eine systematische Benachteiligung von spezifischen „Randgruppen“ auf. Insbesondere Personen mit niedrigem Schulabschluss gelingt es nur bedingt, sich langfristig in Berufausbildung und Arbeitsmarkt zu etablieren. Ähnlich kritische Ergebnisse zeigen sich für junge Menschen mit Migrationshintergrund. Der dadurch verursachte Mangel an Fachkräften stellt nicht nur volkswirtschaftlich ein Problem dar. Zudem werden einzelne Bevölkerungsgruppen dadurch systematisch von den positiven Effekten von Bildung ausgeschlossen, etwa hinsichtlich einer selbstverantwortlichen Gestaltung des Lebenslaufs oder, wie gezeigt werden konnte, auch einer höheren Lebenserwartung in Gesundheit. Mit Blick auf den demografischen Wandel darf sich Politik somit nicht nur darauf beschränken, die Bildung der älteren Bevölkerung zu fördern, sondern muss bereits in den vorangehenden frühen Phasen von Bildung und Ausbildung die Diskriminierung spezifischer Gruppen von „Bildungsverlierern“ abbauen, etwa durch spezifischer Integrationsund Fördermaßnahmen für benachteiligte Gruppen und Geringqualifizierte.

5. Auch die demografische und sozialwissenschaftliche Forschung muss sich konzeptionell und methodisch den gewandelten sozioökonomischen Bedingungen anpassen. Eine gleichmäßigere Verteilung von Arbeit und Bildung über den Lebenslauf des Einzelnen und über die Bevölkerungsgruppen hinweg sollte dabei auch in der sozialwissenschaftlichen und demografischen Forschung stärker thematisiert werden (Wheelock \& Vail 1999; Blekesaune \& Quadagno 2003; Marshall et al. 2003; Lee \& Goldstein 2003). Geschlossene Erwerbsbiografien gibt es immer seltener, berufliche Entwicklungswege sind mit größeren Unsicherheiten und Kontingenzen behaftet. Diesem Umstand muss sich in Zukunft auch die Forschung stärker zuwenden: Marshall et al.(2003) weisen darauf hin, dass Forschung und politische Strategien sich vor allem auf verschiedene Lebensstadien und ihre Übergänge konzentrieren. Aber gerade die Interaktionen verschiedener alters- und phasenspezifischer Phänomene verdienen Aufmerksamkeit, ebenso wie die Betrachtung des gesamten Lebenslaufs.

Das vergangene Jahrhundert war bestimmt von der Frage nach einer gerechten Umverteilung von Wohlstand. In dem nun angebrochenen Jahrhundert wird es dagegen mehr um die Umverteilung von Arbeit gehen (Vaupel 2000; Vaupel \& Loichinger 2006). Es handelt sich dabei nicht nur um eine Lifestyle-Frage, ob junge Eltern in Teilzeit arbeiten oder Ältere gleiche Chancen auf dem Arbeitsmarkt haben wie Jüngere. Aufgrund des demografischen Wandels entstehen ökonomische Notwendigkeiten, die letztlich dazu führen werden, dass sich die Frage nach der Vereinbarkeit von Familie und Beruf und nach der Beteiligung Älterer am Erwerbsleben viel grundsätzlicher stellen wird. Ein Umdenken ist folglich in vielen Bereichen der Gesellschaft ebenso wie bei der individuellen Gestaltung des Lebens geboten.

Danksagungen: Wir danken Kristín G. v. Kistowski für ihre Mitarbeit an diesem Beitrag sowie Roland Rau, Elke Loichinger, Harald Wilkoszewski, Annette Baudisch, Katharina Frosch, Juliane Steinberg, Heiner Maier und Andreas Edel für kritische Kommentare sowie Silvia Leek und Stefanie Moll für die Unterstützung bei den Grafiken. 


\section{Anmerkungen}

1 Die mittlere Variante (1-W1) geht von einem etwa konstanten Geburtenniveau, einer jährlichen Zuwanderung von 100.000 und einem vergleichsweise geringen Anstieg der Lebenserwartung aus. In der Basisannahme ergibt sich für das Jahr 2050 für Männer eine durchschnittliche Lebenserwartung bei Geburt von 83,5 Jahren und für Frauen von 88,0 Jahren. Das ist ein Zuwachs von 7,6 beziehungsweise 6,5 Jahren im Vergleich zur Lebenserwartung in Deutschland 2002/2004. Die Differenz in der Lebenserwartung von Männern bzw. Frauen verringert sich bis 2050 von 5,6 auf 4,5 Jahre (Statistisches Bundesamt 2006).

2 Nach den Periodensterbetafeln für das Deutsche Reich. Quelle: Human Life-Table Database.

3 In den neuen Bundesländern kommt hinzu, dass die Abwanderungswelle vor allem junger Menschen und das Geburtentief der 1990er-Jahre im kommenden Jahrzehnt einen „Echoeffekt" auslösen werden, welcher die Bevölkerungsalterung weiter verschärft.

4 Ein Beispiel stellt etwa das alternde, schrumpfende Erwerbspotenzial dar: Ist die erwerbsfähige Bevölkerung bereits geschrumpft, so kann nur dann das Potenzial derer genutzt werden, die bislang wenig in den Arbeitsmarkt integriert waren, wenn diese zuvor ausreichend Qualifikationen erwerben konnten.

5 Beispielsweise zeigen sich bei Krebserkrankungen und bei Erkrankungen der Atemwege umgekehrte Trends: Bestehende Unterschiede nach Bildungshöhe sind über den Zehnjahresabstand der Beobachtung fast verschwunden (Rau et al. 2008).

6 Ein Beispiel mag das Rauchverhalten von Frauen liefern. In Dänemark hat sich beispielsweise die Lebenserwartung für Frauen nur sehr schleppend entwickelt. Insbesondere viele zwischen den Kriegen geborene Frauen erwiesen sich im Vergleich zu vorangegangenen Generationen als starke, langjährige Raucherinnen - mit deutlicher Auswirkung auf die Entwicklung der Lebenserwartung (Jacobsen et al. 2002). Die nachfolgend geborenen Frauen weichen jedoch in ihrem durchschnittlichen Rauchverhalten von diesen Kohorten ab, so dass ein Aufholen zu beobachten ist. Im europäischen Vergleich steht Dänemark inzwischen sogar ausgesprochen gut da (Jagger et al. 2008). Kohortenweise auftretende Änderungen im Verhaltensmuster bestätigt auch Schwarz (2005) für österreichische Frauen: Er beobachtet einen „Crossover“ im Rauchverhalten nach sozialer Schichtung: Unter Frauen, die jünger als 45 Jahre sind, rauchen solche mit niedrigem Bildungsabschluss mehr, während unter älteren Frauen vor allem diejenigen mit höherem Abschluss Raucherinnen sind.

7 Der Wert fiel so niedrig aus, weil nur 44\% der Menschen in Deutschland überhaupt erwerbstätig waren.

8 Innerhalb der Bevölkerung im Erwerbsalter (20 bis 65 Jahre) sinkt der Anteil der mittleren Altersgruppe (30 bis 49 Jahre) ausgehend von 50\% im Jahr 2005 innerhalb von 15 Jahren auf $42 \%$, während der Anteil der älteren Menschen im Erwerbsalter ( 50 bis 65 Jahre) von $30 \%$ auf $40 \%$ steigt. Anschließend wird die Erwerbsbevölkerung zusätzlich schrumpfen - selbst bei der optimistischen Annahme von 200.000 Zuwanderern jährlich sinkt sie bis zum Jahr 2050 auf $78 \%$ des Niveaus von 2005 (Statistisches Bundesamt 2006).

9 Die Literatur liefert sogar Hinweise auf einen fehlenden oder nur geringen Zusammenhang zwischen Alter und Produktivität (Dittmann-Kohli \& van der Heijden 1996; Lehr 2000).

Open Access: Dieser Artikel unterliegt den Bedingungen der Creative Commons Attribution Noncommercial License. Dadurch sind die nichtkommerzielle Nutzung, Verteilung und Reproduktion erlaubt, sofern der/die Originalautor/en und die Quelle angegeben sind. 


\section{Literatur}

Allmendinger, J. (2000). Wandel von Erwerbs- und Lebensverläufen und die Ungleichheit zwischen den Geschlechtern im Alterseinkommen. In W. Schmähl \& K. Michaelis (Hrsg.), Alterssicherung von Frauen: Leitbilder, gesellschaftlicher Wandel und Reformen (S. 61-80). Wiesbaden: Westdeutscher Verlag.

Allmendinger, J., \& Ebner, C. (2006). Arbeitsmarkt und demografischer Wandel: Die Zukunft der Beschäftigung in Deutschland. Zeitschrift für Arbeits- und Organisationspsychologie, 50(4), 227-239.

Allmendinger, J., Dressel, K., \& Ebner, C. (2006). Zum Verhältnis von Demografie, Qualifikation und Frauenerwerbstätigkeit. In Ministerium für Generationen, Frauen, Familie und Integration NRW (Hrsg.), Demografischer Wandel: Die Stadt, die Frauen und die Zukunft (S. 161-172). Köln: MGFFI NRW.

Arnds, P., \& Bonin, H. (2003). Frühverrentung in Deutschland: Ökonomische Anreize und institutionelle Strukturen. In M. Herfurth, M. Kohli \& K. F. Zimmermann (Hrsg.), Arbeit in einer alternden Gesellschaft (S. 65-91). Opladen: Leske + Budrich.

Auer, P., \& Fortuny, M. (2000). Aging of the Labour Force in OECD Countries: Economic and Social Consequences (ILO Employment Paper 2000/2). Geneva: International Labour Office.

Autorengruppe Bildungsberichterstattung (2008). Bildung in Deutschland 2008. Ein indikatorengestützter Bericht mit einer Analyse zu Übergängen im Anschluss an den Sekundarbereich I. Bielefeld: W. Bertelsmann.

Barford, A., Dorling, D., Smith, G., \& Shaw, M. (2006). Life expectancy: Women now on top everywhere. During 2006, even in the poorest countries, women can expect to outlive men. British Medical Journal, 332, 808.

Baethge, M., Solga, H., \& Wieck, M. (2007). Berufsbildung im Umbruch: Signale eines überfälligen Aufbruchs. Berlin: Friedrich-Ebert-Stiftung.

Blekesaune, M., \& Quadagno, J. (2003). Public attitudes towards welfare state policies - A comparative analysis of 24 Nations. European Sociological Review, 19, 415-427.

Blossfeld, H.-P. (2003). Globalization, social inequality and the role of country-specific institutions. In P. Conceicao, M. V. Heitor, \& B.-A. Lundvall (Eds.), Innovation, competence building and social cohesion in Europe. Towards a learning society (pp. 303-334). Cheltenham, UK: Edward Elgar.

Blossfeld, H.-P., \& Hofmeister, H. (2006). Globalization, uncertainty and women's careers: An international comparison. Cheltenham, UK: Edward Elgar.

Blossfeld, H.-P., Buchholz, S., \& Hofäcker, D. (2006). Globalization, uncertainty and late careers in society. London: Routledge.

Blossfeld, H.-P., Drobnič, S., \& Rohwer, G. (2001). Spouses' employment careers in West Germany. In H.-P. Blossfeld \& S. Drobnič (Hrsg.), Careers of couples in contemporary society: From male breadwinner to dual-earner families (pp. 53-76). Oxford: Oxford University Press.

Blossfeld, H.-P., Klijzing, E., Mills, M., \& Kurz, K. (Eds.). (2005). Globalization, uncertainty and youth in society. London: Routledge.

Blossfeld, H-P., Kurz, K., Buchholz, S., \& Bukodi, S. (Eds.). (2009). Young workers, globalization and the labor market: Comparing early working life in eleven countries. Cheltenham, UK: Edward Elgar.

Börsch-Supan, A. (2003). Labor market effects of population aging. Labor, 17, 5-44.

Börsch-Supan, A., Düzgun, I., \& Weiss, M. (2005). Altern und Produktivität: Zum Stand der Forschung, Mannheim: Universität.

Buchholz, S. (2008). Die Flexibilisierung des Erwerbsverlaufs. Eine Analyse von Einstiegs- und Ausstiegsprozessen in Ost- und Westdeutschland. Wiesbaden: VS Verlag für Sozialwissenschaften. 
Buchholz, S., \& Grunow, D. (2006). Women's employment in West Germany. In H.-P. Blossfeld $\&$ H. Hofmeister (Eds.), Globalization, uncertainty and women's careers: An international comparison (pp. 61-83). Cheltenham, UK: Edward Elgar.

Crimmins, E., Reynolds, S. L., \& Saito, Y. (1999). Trends in health and ability to work among the older working-age population. Journal of Gerontology, 54B(1), 31-40.

Dittmann-Kohli, F., \& van der Heijden, B. (1996). Leistungsfähigkeit älterer Arbeitnehmer - interne und externe Faktoren. Zeitschrift für Gerontologie, 29, 323-327.

Doblhammer, G. (2006). Das Alter ist weiblich. Demographie der weiblichen Bevölkerung. Der Gynäkologe 30(5), 346-353.

Doblhammer, G., \& Ziegler, U. (2006). Future elderly living conditions in Europe: Demographic insights. In G. M. Backes, V. Lasch, \& K. Reimann K. (Hrsg.), Gender, health and ageing. European perspectives (pp. 267-292). Wiesbaden: VS Verlag für Sozialwissenschaften.

Doblhammer, G., Muth, E., \& Kruse, A. (2008). Lebenserwartung in Deutschland: Trends, Prognose, Risikofaktoren und der Einfluss ausgewählter Medizininnovationen. Projektbericht für den Verband forschender Arzneimittelhersteller (S. 157). Rostocker Zentrum zur Erforschung des Demografischen Wandels. Rostock: Rostocker Zentrum zur Erforschung des demografischen Wandels.

Doblhammer, G., Rau, R., \& Kytir, J. (2005). Trends in educational and occupational differentials in all-cause mortality in Austria between 1981/82 and 1991/92. Wiener Klinische Wochenschrift, 117, 468-479.

Dorbritz, J., Höhn, C., \& Naderi, R. (2005). The demographic future of Europe - Facts, figures, policies. Ergebnisse der Population Policy Acceptance Study (DIALOG-PPAS). Stuttgart \& Wiesbaden: Robert Bosch Stiftung, Bundesinstitut für Bevölkerungsforschung.

Duschek, K.-J., \& Wirth, H. (2005). Kinderlosigkeit von Frauen im wohl : Spiegel des Mikrozensus. Eine Kohortenanalyse der Mikrozensen 1987-2003. Wirtschaft und Statistik, 8, 800-820.

Ebbinghaus, B. (2005). Vom Stilllegen von Arbeit zum lebenslangen Lernen. Das überfällige Ende der Frühverrentung in Europa, Japan und den USA. In Max-Planck-Institut für Gesellschaftsforschung (Hrsg.), MPIfG Jahrbuch 2003-2004 (S. 37-42). Köln: Max-Planck-Institut für Gesellschaftsforschung.

Eurostat (2007). Population and social conditions. http://epp.eurostat.ec.europa.eu/portal/ page? pageid $=0,1136184,0 \_45572592 \&$ dad=portal\&_schema=PORTAL

Frejka, T., \& Sobotka, T. (2008). Fertility in Europe: Diverse, delayed and below replacement. Demographic Research, 19 (Special Collection 7: Childbearing Trends and Policies in Euope), 15-46. (www.demographic-research.org/special/7/)

Frosch, K. (2007). Zu alt für einen neuen Job? Altersspezifische Wiederbeschäftigungschancen nach Verlust des Arbeitsplatzes. Wirtschaftspolitische Blätter, 54(4), 641-656.

Hackmann, T., \& Moog, S. (2008). Die Auswirkungen der steigenden Lebenserwartung auf die Prävalenz der Pflegebedürftigkeit in Deutschland. Zeitschrift für Versicherungswissenschaft, 98(1), 73-89.

Heinz, W. R. (2003). From work trajectories to negotiated careers: The contingent work life course. In J. T. Mortimer \& M. J. Shanahan. (Eds.), Handbook of the life course (pp. 185-204). New York: Kluwer Academic/Plenum.

Hoem, J. M., Neyer, G., \& Andersson, G. (2006). Education and childlessness: The relationship between educational field, educational level, and childlessness among Swedish women born in 1955-1959. Demographic Research, 14(15), 331-380.

Hofäcker, D. (in press). Older workers under Globalization: An international comparison of retirement and late-career patterns among older workers in Western industrialized countries. Cheltenham, UK: Edward Elgar. 
Hofäcker, D., \& Pollnerová, S. (2006). Late careers and career exits. An international comparison of trends and institutional background patterns. In H.-P. Blossfeld, S. Buchholz \& D. Hofäcker (Eds.), Globalization, uncertainty and late careers in society (pp. 25-53). London: Routledge.

Hofäcker, D., Buchholz, S., \& Blossfeld, H.-P. (2007). Globalisierung, struktureller Wandel und die Veränderung später Erwerbskarrieren - Deutschland im internationalen Vergleich. Deutsche Rentenversicherung, 62(4-5), 197-215.

Hofäcker, D., Schröder, H., \& Müller-Camen, M. (2008). In Deutschland mehr Vorbehalte gegenüber älteren Arbeitnehmern: ein deutsch-britischer Vergleich. Informationsdienst soziale Indikatoren (ISI), 41, 7-11.

Hradil, S. (2006). Was prägt das Krankheitsrisiko: Schicht, Lage, Lebensstil? In M. Richter \& K. Hurrelmann (Hrsg.), Gesundheitliche Ungleichheit. Grundlagen, Probleme, Perspektiven (S. 33-52). Wiesbaden: VS Verlag für Sozialwissenschaften.

Jacobsen, R., Keiding, N., \& Lynge, E. (2002). Long term mortality trends behind low life expectancy of Danish women. Journal of Epidemiology and Community Health, 56, 205-208.

Jacobzone, S., Cambois, E., \& Robine, J. M. (2000). Is the health of older persons in OECD Countries improving fast enough to compensate for population ageing? OECD Economic Studies, 30, 149-190.

Jagger, C., Gillies, C., Moscone, F., Cambois, E., Oyen, H. V., Nusselder, W., et al. (2008). Inequalities in healthy life years in the 25 countries of the European Union in 2005: a cross-national meta-regression analysis. The Lancet, 372, 2124-2131.

Kohli, M. (1985). Die Institutionalisierung des Lebenslaufs: Historische Befunde und theoretische Argumente. Kölner Zeitschrift für Soziologie und Sozialpsychologie, 37, 1-29.

Konietzka, D., \& Kreyenfeld, M. (2007). Die Analyse von Kinderlosigkeit in Deutschland. Dimensionen - Daten - Probleme. In D. Konietzka \& M. Kreyenfeld. (Hrsg.), Ein Leben ohne Kinder. Kinderlosigkeit in Deutschland (S. 11-41). Wiesbaden: VS Verlag für Sozialwissenschaften.

Koskinen, S. (2003). Commentary: Is there a common background behind growing inequalities in mortality in Western European countries? International Journal of Epidemiology, 32, $838-839$.

Kunst, A. E., Bos, V., Andersen, O., Borgan, J.-K., Cardano, M., Costa, G., Harding, S., et al. (2004). Monitoring of trends in socioeconomic inequalities in mortality: Experiences from a European project. Demographic Research, Special Collection, 2, 229-253.

Lee, R. D., \& Goldstein, J. R. (2003). Rescaling the life cycle: Longevity and proportionality. In J. R. Carey \& S. Tuljapurkar (Eds.), Life span: Evolutionary, ecological, and demographic perspectives (pp. 183-207). Supplement to Vol. 29 of Population and Development Review. New York: Population Council.

Lehr, U. (2000). Psychologie des Alterns. Wiebelsheim: Quelle \& Meyer.

Lutz, W., Sanderson, W., \& Scherbov, S. (2008). The coming acceleration of global population aging [Letter]. Nature, 7179, 716-719.

Lynch, J. W., Kaplan, G. A., \& Salonen, J. T. (1997). Why do poor people behave poorly? Variation in adult health behaviours and psychosocial characteristics by stages of the socioeconomic life course. Social Science and Medicine, 44, 809-819.

Mackenbach, J. P., Bos, V., Anderson, O., Cardano, M., Costa, G., Harding, S., et al. (2003). Widening socioeconomic inequalities in mortality in six Western European countries. International Journal of Epidemiology, 32, 830-837.

Marmot, M. (2005). Social determinants of health inequalities. The Lancet, 365, 1099-1104.

Marshall, V. W., \& Müller, M. M. (2002). Rethinking social policy for an ageing workforce and society: Insights from the life course perspective (CPRN Discussion Paper, No. W/18). Ottawa: Canadian Policy Research Networks.

Marshall, V. W., Heinz, W. R., Kruger, H., \& Verma, A. (2003). Restructuring work and the life course. Toronto: University of Toronto Press. 
Naegele, G., Barkholdt, C., De Vroom, B., Andersen, J. G., \& Krämer, K. (2003). A new organisation of time over working life. Report published by the European foundation for the improvement of living and working conditions. Luxemburg: Office for official publications of the European Communities.

Neyer, G., \& Andersson, G. (2004). Contemporary research on European fertility. Demographic Research, Special issue 3. (www.demographic-research.org/special/3)

Neyer, G., \& Hoem, J. M. (2008). Education and permanent childlessness: Austria vs. Sweden. A research note. In J. Surkyn, J. van Bavel \& P. Deboosere (Eds.), Demographic challenges for the 21st century. A tribute to the continuing endeavors of Ron Lesthaeghe in the field of demography (pp. 91-112). Brussels: VUB, Academia Press.

Oeppen, J., \& Vaupel, J. W. (2002). Broken limits to life expectancy. Science, 296, 1029-1031.

OECD (Organisation of Economic Co-Operation and Development) (1999). Training of adult workers in OECD countries: Measurement and analysis, OECD employment outlook 1999, http:// www.oecd.org/dataoecd/9/5/2079990.pdf. Zugriff 24. Juli 2009.

OECD (Organisation of Economic Co-Operation and Development) (2001). Reforms for an ageing society. Paris: OECD.

OECD (Organisation of Economic Co-Operation and Development) (2007). OECD employment outlook 2007, http://www.oecd.org/document/38/0,3343,en_2649_33927_36936230_1_1_1_ 1,00.html. Zugriff 24. Juli 2009.

OECD (Organisation of Economic Co-Operation and Development) (2008). Education at a glance. Paris: OECD.

PRB (Population Reference Bureau) (2008). World population data sheet, http://www.prb.org/Publications/Datasheets/2008/2008wpds.aspx. Zugriff 24. Juli 2009.

Rau, R., Doblhammer, G., Canudas-Romo, V., \& Zhen, Z. (2008). Cause-of-death contributions to educational inequalities in mortality in Austria between 1981/1982 and 1991/1992. European Journal of Population, 24, 265-286.

Rau, R., Soroko, E., Jasilionis, D., \& Vaupel, J. W. (2006). 10 years after Kannisto: Further evidence for mortality decline at advanced ages in developed countries (MPIDR working paper WP-2006-033), http://www.demogr.mpg.de/papers/working/wp-2006-033.pdf. Zugriff 24. Juli 2009.

Reday-Mulvey, G. (2005). Working past 60: Key policies and practices in Europe. Basingstoke: Palgrave Macmillan.

Reinberg, A., \& Hummel, M. (2004). Fachkräftemangel bedroht Wettbewerbsfähigkeit der deutschen Wirtschaft. Aus Politik und Zeitgeschichte B, 28, 3-10.

Richter, M., \& Hurrelmann, K. (2006). Gesundheitliche Ungleichheit: Ausgangsfragen und Herausforderungen. In M. Richter \& K. Hurrelmann (Hrsg.), Gesundheitliche Ungleichheit. Grundlagen, Probleme, Perspektiven (S. 11-31). Wiesbaden: VS Verlag für Sozialwissenschaften.

Riley, J. (2001). Rising life expectancy: A global history. Cambridge: Cambridge University Press.

Schröder, H., Hofäcker, D., \& Müller-Camen, M. (2009). HRM and the employment of older workers: Germany and Britain compared. International Journal of Human Resource Management and Development, 9(2/3), 162-179.

Schwarz, F. (2005). Widening educational inequalities in mortality: An analysis for Austria with international comparisons (VID Working Paper 07/2005). Vienna: Vienna Institute of Demography, http://hw.oeaw.ac.at/0xc1aa500d_0'0017f0d9.pdf. Zugriff 24. Juli 2009.

Sobotka, T. (2004). Postponement of childbearing and low fertility in Europe. Amsterdam: Dutch University Press.

Statistisches Bundesamt (2006). 11. koordinierte Bevölkerungsvorausberechnung. Annahmen und Ergebnisse, http://www.destatis.de/jetspeed/portal/ cms/Sites/destatis/Internet/DE/Presse/ $\mathrm{pk} / 2006 /$ Bevoelkerungsentwicklung/Annahmen__und_Ergebnisse,property=file.pdf. Zugriff 24. Juli 2009. 
United Nations (2007). World population ageing. New York: United Nations Department of Economic and Social Affairs, Population Division.

Valkonen, T. (1989). Adult mortality and level of education. A comparison of six countries. In J. Fox (Ed.), Health inequalities in European countries (pp. 142-162). Aldershot: Gower.

Valkonen, T. (2006). Social inequalities in mortality. In G. Caselli, J. Vallin \& G. Wunsch. (Eds.), Demography. Analysis and synthesis (Vol. II, Chap. 54, pp. 195-206). Amsterdam: Elsevier.

Vallin, J., \& Meslé, F. (2001). Trends in mortality in Europe since 1950: Age-, sex- and causespecific mortality. In J. Vallin, F. Meslé \& T. Valkonen (Eds.), Trends in mortality and differential mortality: Vol. 36. Population studies (pp. 31-184). Strasbourg: Council of Europe Publishing.

Vaupel, J. W. (2000). Setting the stage: A generation of centenarians? The Washington Quarterly, 2(2), 197-200.

Vaupel, J. W. (2006). Prospects for longevity (MPIDR Working Paper 2006-037), http://www. demogr.mpg.de/en/publications/working.htm. Zugriff 24. Juli 2009.

Vaupel, J. W., \& Kistowski, K. G. v. (2007). Die Plastizität menschlicher Lebenserwartung und ihre Konsequenzen. In P. Gruss. (Hrsg.), Die Zukunft des Alterns (S. 51-78). München: Beck.

Vaupel, J. W., \& Loichinger, E. (2006). Redistributing work in aging Europe. Science, 312, 1911 1913.

Vaupel, J. W., \& Owen, J. M. (1986). Anna's life expectancy. Journal of Policy Analysis and Management, 5(2), 383-389.

Vaupel, J. W., Schnabel, S., Kistowski, K. G. v, \& Gampe, J. (2006). Möglichkeiten und Grenzen demographischer Prognosen. In T. Petersen (Hrsg.), Demographiemonitor, Bd. 2: Handlungsoptionen im demographischen Wandel (S. 35-59). Gütersloh: Bertelsmann Stiftung.

vbw (Vereinigung der Bayerischen Wirtschaft e.V.) (Hrsg.) (2008). Bildungsrisiken und Chancen im Globalisierungsprozess. Jahresgutachten 2008. Wiesbaden: VS Verlag für Sozialwissenschaften.

vbw (Vereinigung der Bayerischen Wirtschaft e.V.) (Hrsg.) (2009). Geschlechterdifferenzen im Bildungssystem. Jahresgutachten 2009. Wiesbaden: VS Verlag für Sozialwissenschaften.

Wheelock, J., \& Vail, J. (Eds.) (1999). Work and idleness: The political economy of full employment. Berlin: Springer.

World Bank (1994). Averting the old age crisis. Oxford: Oxford University Press. 